\title{
Evolutionary game model of group choice dilemmas on hypergraphs
}

\author{
Andrea Civilini, ${ }^{1}$ Nejat Anbarci, ${ }^{2}$ and Vito Latora ${ }^{1,3,4}$ \\ ${ }^{1}$ School of Mathematical Sciences, Queen Mary University of London, London E1 4NS, United Kingdom \\ ${ }^{2}$ Department of Economics and Finance, Durham University, Durham DH1 3LB, United Kingdom \\ ${ }^{3}$ Dipartimento di Fisica ed Astronomia, Università di Catania and INFN, Catania I-95123, Italy \\ ${ }^{4}$ Complexity Science Hub Vienna, A-1080 Vienna, Austria
}

\begin{abstract}
We introduce an evolutionary game on hypergraphs in which decisions between a risky alternative and a safe one are taken in social groups of different sizes. The model naturally reproduces choice shifts, namely the differences between the preference of individual decision makers and the consensual choice of a group, that have been empirically observed in choice dilemmas. In particular, a deviation from the Nash equilibrium towards the risky strategy occurs when the dynamics takes place on heterogeneous hypergraphs. These results can explain the emergence of irrational herding and radical behaviours in social groups.
\end{abstract}

Choice dilemmas describe the most general situations in which decision makers are faced with an alternative between two possibilities: a riskier strategy, that either brings a high reward, with a probability $w_{p}$, or a low one, with probability $1-w_{p}$, and a safer strategy with an intermediate reward [1-3]. Expected Utility Theory and its strategic version Game Theory assume that rational decision makers always act to maximize their private utilities/payoffs [4]. However, over the years a series of empirical evidences in contrast with the theoretical predictions have clearly pointed out the descriptive limitations of these theories and have undermined their very fundamental assumptions. Alternative explanations of these discrepancies are based on ad hoc behavioural mechanisms in the decision making process [1, 3, 5-13] such as loss aversion [6], risk diffusion [14, 15], rational conformity [16], social facilitation [17] and group polarization $[2,7,18]$, i.e. individual opinion polarization through social interaction processes [19-22] such as persuasive argumentation and social comparison [23]. In particular, two anomalies of great practical relevance arise when decision makers interact in social groups, influencing each other. The first anomaly is the herd behaviour leading to irrational outcomes, observed for example during financial bubbles, where decision makers can follow the opinion of others, apparently disregarding their own interests [1013]. The second anomaly has been empirically observed when decision makers are organized in groups and decide collectively their strategy [2, 3, 7, 24] (a precious source of insights and experimental data is offered by the studies on trial juries conducted in the Seventies [25-29], see Supplemental Material, SM [30]). In this context a choice shift effect emerges, in which the average opinion of individual decision makers is exacerbated when they act in group $[1,7,18]$. All the existing theories for herd behaviour and choice shift have some major drawbacks. First of all, no existing theory is able to reproduce both anomalies at the same time. Secondly, existing models consider isolated social groups of fixed size, neglecting the presence of groups of heterogeneous sizes and the nested hierarchical structure of real social systems, which are known to play a key role in the emergence of critical phenomena [35-37]. Finally, the existing models focus on either one or the other of the two main aspects of group decision: information spreading $[16,17,38]$ and the aggregation of preferences $[3,7,39]$.

In this Letter, we propose to use evolutionary game theory on hypergraphs to model group choice dilemmas. Higher-order interactions have recently been shown to influence dynamical processes [40], including social contagion [41] and evolutionary games [42, 43]. Our model can be seen as a generalization to higher-order interactions of anti-coordination pairwise games, such as the game of Chicken [44], in which the decision makers are organized in groups of different sizes and each decision maker can participate to a variable number of groups. Differently from all previous models, our game dynamics describes, at same time, how opinions spread according to an imitation process and how they are aggregated by the members of a group to determine the group strategy. We show that, due to this, group choice shifts emerge naturally in our model, and we explain how they depend on the mechanisms of preference aggregation and on the structure of the hypergraph. In particular, when implemented on heterogeneously structured populations, our model predicts the spontaneous emergence of irrational herd behaviours towards the riskier strategy.

Model. A population of $M$ interacting decision makers is modelled as the nodes of a hypergraph, whose $N$ hyperedges describe the interactions in groups of two or more agents $[40,45]$. The hypergraph can be represented by a $M \times N$ adjacency matrix $A$, whose entry $a_{i}^{(g)}$ is equal to 1 if the agent $i$ is in group $g$, or is zero otherwise. The hyperdegree of agent $i, k_{i}=\sum_{g \in N} a_{i}^{(g)}$, and the size of group $g, q^{(g)}=\sum_{i \in M} a_{i}^{(g)}$, represent respectively the number of groups in which agent $i$ takes part, and the number of members of group $g$. Let $Q(q)$ and $K(k)$ be the probability distributions of group sizes and agent hyperdegrees respectively [46]. In particular, we focus on power-law distributions $Q(q) \sim q^{-\lambda}$ and $K(k) \sim k^{-\nu}$. In fact, many real-world group sizes are 
power-law distributed: scientific teams [47, 48], firms [49], human settlements/cities [50] and also groups of animals $[51,52]$. Moreover, it has been shown that also the number of social activities/groups in which a person is involved (i.e. the hyperdegree) follows long-tailed distributions $[47,53,54]$. Given the probability of success of a risky activity $0 \leq w_{p} \leq 1$, each agent can be in one of two states: $\mathbf{s}$ (safe) or $\mathbf{t}$ (tempted). If in state $\mathbf{s}$, the agent prefers not to participate to the risky activity. Instead if in state $\mathbf{t}$, agent is willing to share with the other group members the cost $C$ to attempt this risky strategy. The strategy of a group, namely the decision to participate to the risky activity (group strategy $\mathbf{T}$ ) or not (strategy $\mathbf{S}$ ), depends on the states of the group members and on the way in which these states/personal opinions are aggregated. We have considered different group decision schemes, i.e. rules of opinion aggregation, ranging from simple majority (where the group adopts the strategy preferred by at least half of the group members +1 ), to two-third majority, and proportionality [55]. If we indicate with $C$ the cost for the group to adopt a risky strategy, and with $T$ and $S$ the rewards, respectively in the case of success or failure of the risky activity, then we assume the following group payoffs $\pi^{(g)}$ :

$$
\begin{aligned}
\pi^{(g)}(\mathbf{S}) & =S \\
\pi^{(g)}(\mathbf{T}) & =\left\{\begin{array}{l}
W:=T-C, \text { with probability } w_{p} \\
L:=S-C, \text { with probability } 1-w_{p}
\end{array}\right.
\end{aligned}
$$

associated with the group strategies. Given $0<C \leq$ $S<T$, it follows that $L<S<W$ and then we are in a classical choice dilemma scenario, where the risky choice $\mathbf{T}$ brings a high payoff $W$ (with probability $w_{p}$ ) or a low payoff $L$ (with $1-w_{p}$ ), while the safe action $\mathbf{S}$ guarantees an intermediate payoff $S$. Let us define as $z=N_{T} / N$ the fraction of groups with strategy $\mathbf{T}$. In our model at each time step the $z N$ groups with strategy $\mathbf{T}$ are in competition for a fixed share $W$ of the total reward $\theta N W$, with $0<\theta<1$. As a consequence, the probability of success $w_{p}$ of a risky activity is modelled as a non-decreasing function of the total number of shares $\theta N$ per group with strategy $\mathbf{T}: w_{p}=f\left(\frac{\theta}{z}\right)$. Moreover, in our model the successful groups are selected uniformly at random among the $z N$ groups with strategy $\mathbf{T}$. Hence, the higher is $z$, the fraction of player with strategy $\mathbf{T}$, the lower is the chance of high reward (i.e. actions $\mathbf{T}$ are strategic substitutes [56]). We show in the SM [30] that, when played by two groups of equal size, our game is equivalent to the pairwise game of Chicken [57]. To keep the model as general as possible, we allow the cost $C$ to be a non-increasing function $C\left(r^{(g)}\right)=\left(r^{(g)}\right)^{-1}$ of a group resource $r^{(g)} \in \mathbb{R}^{+}$. The idea is that the more resource a group has, the lower the cost for attempting the risky activity and the risk perception are [14, 15]. To define the resource of a group, we assume that each agent $i$ is given an individual resource $r_{i}=r_{\min } k_{i}$ that the agent splits equally among the $k_{i}$ groups to which it participates. This is a realistic assumption, as the greater is the resource of an agent the larger will be on average the number of activities it is involved [58]. Moreover, this means that agent $i$ invests in each of its $k_{i}$ activities an amount of resource $r_{i}^{(g)}=r_{\text {min }}$. Consequently, the total resource $r^{(g)}$ of a group $g$ is a function of its size $q^{(g)}$ :

$$
r^{(g)}=\left(\sum_{i \in g} r_{i}^{(g)}\right)^{\beta}=\left(r_{\min } q^{(g)}\right)^{\beta}
$$

where the exponent $\beta \geq 0$ takes into account possible nonlinear synergistic effects raising from the interaction among group members [59]. In particular, for $\beta>1$ the interaction among agents leads to a superlinear scaling of the group resource with the group size. To determine the payoff of the individual agents, we simply assume that the payoff of a group in Eq. (1) is equally shared among its group members. Then, the payoff of agent $i$ is defined as the sum of the returns from all the groups in which it is involved: $\pi_{i}=\sum_{g \mid i \in g} \pi^{(g)} /\left(q^{(g)}\right)^{\zeta}$. The exponent $0 \leq \zeta \leq 1$ allows to tune the way in which group members benefit from the group payoff. For example, in contexts where the payoff represents a material or countable quantity (e.g. a cash prize), $\zeta=1$ implies that the group payoff is equally split among the group members and the total group payoff is conserved. In the limit $\zeta=0$ instead each group member earns the full payoff coming from the group [60]. In the evolutionary dynamics of our model, we assume perfect rationality of the agents, meaning that the agent states are updated in time according to a stochastic dynamics where each agent tries to imitate the fittest neighbour [61]. Namely, at each time step, a focal individual $i$ is selected at random in the population. A second individual $j$, that we call reference, is randomly selected among the co-members of the focal individual. Then, the probability $p_{i j}$ for the decision maker $i$ to adopt the state of the decision maker $j$ is defined as a growing function of the payoffs difference between the two individuals: $p_{i j}=g\left(\pi_{j}-\pi_{i}\right)$. The co-membership network we consider is obtained as the projection of the hypergraph on the set of nodes representing group members (two nodes are linked if they are co-members in at least one group). We denote as $h_{i}$ the degree of node $i$ on the co-membership network, i.e. the number of co-members of agent $i$, and as $H(h)$ its distribution (see SM [30]). In particular, we verified that for realistic heterogeneously distributed group sizes and hyperdegrees, the resulting co-membership distribution shows power-law tail $H(h) \sim h^{-\gamma}$, as the one observed in many relevant real-world systems [47, 48, 54].

Results. We have investigated how the presence of groups affects decision making through a series of numerical simulations of the model dynamics performed using the quasi-stationary (QS) approach [62, 63] (see SM [30]). 
The numerical simulations on structured population have been compared to an analytically treatable mean-field version of our model dynamics. We describe the mean-field dynamics of the group strategies directly at the group level, by a coarse-grained approach neglecting the microscopic dynamics of decision making that involves the group members. Let us start defining the fraction $z_{q}$ of groups of size $q$ having adopted strategy $\mathbf{T}: z_{q}:=\frac{N_{T}(q)}{N(q)}$, such that $0 \leq z_{q} \leq 1$. In the limit $N \rightarrow \infty$, the time evolution for $z_{q}$ is described by the following equation $[64,65]$ (see SM [30]):

$$
\begin{aligned}
\frac{d z_{q}}{d t}= & \left(1-z_{q}\right) \sum_{q^{\prime}} Q\left(q^{\prime}\right) z_{q^{\prime}} p_{S T}^{q q^{\prime}} \\
& -z_{q} \sum_{q^{\prime}} Q\left(q^{\prime}\right)\left(1-z_{q^{\prime}}\right) p_{T S}^{q q^{\prime}}
\end{aligned}
$$

where $p_{S T}^{q q^{\prime}}$ and $p_{T S}^{q q^{\prime}}$ are respectively the transition probabilities from strategy $\mathbf{S}$ to $\mathbf{T}$ and vice versa, given a focal group of size $q$ and a reference group of size $q^{\prime}$. Such transition probabilities can be expressed as functions of the transition probabilities given the group payoffs $S / q^{\zeta}$, $L / q^{\zeta}$ and $W / q^{\zeta}[60]: p_{S T}^{q q^{\prime}}:=\left(1-w_{p}\right) p_{S L}^{q q^{\prime}}+w_{p} p_{S W}^{q q^{\prime}}$ and $p_{T S}^{q q^{\prime}}:=\left(1-w_{p}\right) p_{L S}^{q q^{\prime}}+w_{p} p_{W S}^{q q^{\prime}}$. Under the assumption of statistical independence $P(q \mid T)=Q(q)$, we get an equation for the time evolution of $z:=\sum_{q} Q(q) z_{q}$ the fraction of groups with strategy $\mathbf{T}$ in the whole population:

$$
\frac{d z}{d t}=z(1-z) \mathbb{E}\left[p_{S T}^{q^{\prime} q}-p_{T S}^{q q^{\prime}}\right]
$$

where the expectation value of function $g\left(q, q^{\prime}\right)$ is defined as $\mathbb{E}\left[g\left(q, q^{\prime}\right)\right]:=\sum_{q, q^{\prime}} g\left(q, q^{\prime}\right) Q(q) Q\left(q^{\prime}\right)$. We recognize in Eq. (4) the celebrated Replicator Equation for a pairwise zero-sum symmetric game [66], with a payoff matrix defined by $\pi(T, S)=\mathbb{E}\left[p_{S T}^{q^{\prime} q}-p_{T S}^{q q^{\prime}}\right]$, which depends in this case on $z$ through $w_{p}(z)$. Hence, besides the two absorbing states $z^{*}=0$ and $z^{*}=1$, the dynamics described by Eq. (4) has a third non trivial stationary solution, which is obtained equating to zero the expectation value in Eq. (4). In particular, in the limit of large population $N>>1$, we can consider $q$ continuously distributed according to $Q(q) \sim q^{-\lambda}$ and replace the sums defining the expectation value with integrals. Under the weak selection hypothesis we can write the transition probability from a generic state $i$ to $j$ as a linear function of the payoffs difference (see SM [30]): $p_{i j}=\frac{1}{2}\left[1+\frac{w_{F}}{2}\left(\pi_{j}-\pi_{i}\right)\right]$, where $-1 \leq \frac{w_{F}}{2}\left(\pi_{j}-\pi_{i}\right) \leq 1$. Substituting this expression in $\mathbb{E}\left[p_{S T}^{q^{\prime} q}-p_{T S}^{q q^{\prime}}\right]=0$ and using the definitions of the group payoffs and $w_{p}=f\left(\frac{\theta}{z}\right)$, we find the nontrivial steady state:

$$
z_{t h}^{*}=\frac{\theta}{f^{-1}\left(\frac{\zeta+\lambda-1}{(\zeta+\lambda+\beta-1)(T-S) r_{\min }^{\beta}}\right)}
$$

We notice that the nonlinearity introduced with the exponents $\beta, \zeta$ and $\lambda$ brings just a scale factor $m:=$ $\frac{\zeta+\lambda+\beta-1}{\zeta+\lambda-1}$ in the solution, without changing its functional form. Since $0 \leq z_{t h}^{*} \leq 1$ by definition, we can define a normalized quantity $0 \leq(T-S)^{\prime} \leq 1$, where $(T-S)^{\prime}:=(T-S)\left[m r_{\min }^{\beta} f(\theta)\right]$, and rewrite Eq. (5) simply as: $z_{t h}^{*}=\theta / f^{-1}\left(f(\theta) /(T-S)^{\prime}\right)$. It can be shown (see SM [30]) that this steady state is an evolutionary stable state [67], and therefore a mixed strategy Nash Equilibrium (NE), of the pairwise zero-sum game defined by Eq. (4). For a probability of success inversely proportional to $z, w_{p}=\theta / z$, we can solve the Cauchy problem associated with Eq. (4) to get an analytical expression for $z(t)$ which converges to $z_{t h}^{*}$ for all the initial conditions $0<z_{0}<1$ (see SM [30]). By comparing $z_{t h}^{*}$, the analytical expression for the null-model's nontrivial steady state, to the long term behaviour of the numerical simulations on structured populations, we can evaluate how the social hypergraph's topology influences the dynamics. Fig. 1
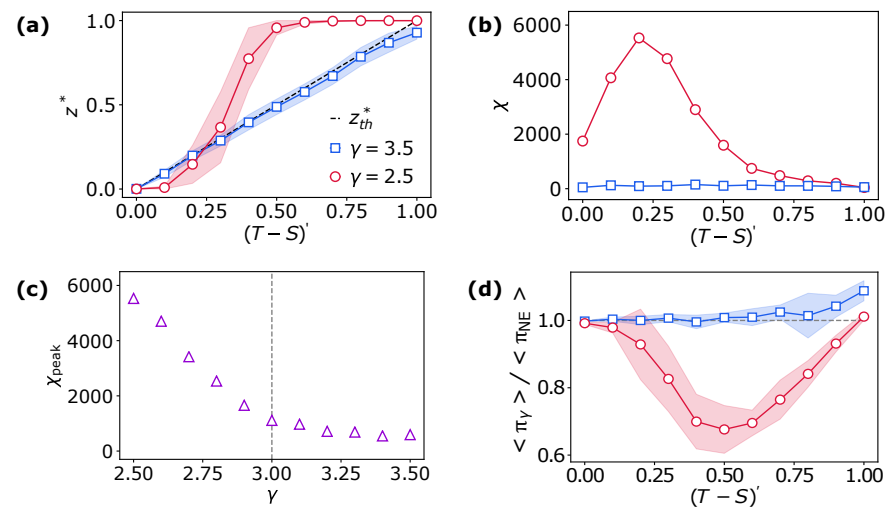

FIG. 1. Long term behaviour of the simulated dynamics on hypergraphs with $H(h) \sim h^{-\gamma}$, for $w_{p}=\theta / z$. (a) For $\gamma=2.5$ we observe a phase transition, while for $\gamma=3.5$ the QS distribution coincides with the NE (dotted line). Points (circles and squares) refer to the median values of $z$. (b) Peak of the susceptibility $\chi$ at the phase transition for $\gamma=2.5$. (c) Peak's height of the susceptibility as a function of the exponent $\gamma$. (d) Average income reduction relative to the NE payoff. Shaded areas show the median absolute deviations. [Parameters: $\beta=0, \zeta=0, \theta=0.01]$.

(a) shows that for hypergraphs characterized by a comembership degree distribution $H(h)$ with a finite second moment (e.g. power-law $H(h) \sim h^{-\gamma}$, with $\gamma=3.5$ ) [68], the numerical simulations are in good agreement with the mean-field solution in Eq. (5), the QS state coinciding with the NE. Instead, when the strategy adoption dynamics takes place on heterogeneous hypergraphs with $H(h) \sim h^{-\gamma}, \gamma<3$ (results shown are for $\gamma=2.5$ ), we found that a phase transition occurs from the absorbing state $z=0$ to a nontrivial stationary state $0<z<1$ that rapidly converges to $z=1$. To better characterize the phase transition we have computed a susceptibility 
function that is commonly used in SIS epidemic models [69]: $\chi=N \frac{\left(\left\langle z^{* 2}\right\rangle-\left\langle z^{*}\right\rangle^{2}\right)}{\left\langle z^{*}\right\rangle}$. Susceptibility functions are specifically designed to peak (diverge in the thermodynamic limit) at the critical value $(T-S)_{c}^{\prime}$ of the order parameter at which the phase transition occurs. The peak of $\chi$ for $\gamma=2.5$ in Fig. 1(b) confirms the occurrence of a phase transition. The plot of the maximum value of the susceptibility as a function of $\gamma$ in Fig. 1 (c) indicates that for $\gamma>3$ the susceptibility is a flat function of $(T-S)^{\prime}$, while for $\gamma<3$ a peak appears, whose height increases as $\gamma$ decreases, pointing out $\gamma=3$ as the threshold value for observing the phase transition. By comparing the average group payoff in the QS state $\left\langle\pi_{\gamma}\right\rangle$ to the expected average payoff at the $\mathrm{NE}\left\langle\pi_{\mathrm{NE}}\right\rangle$ (see SM [30]), we see, Fig. 1 (d), that the QS state for $\gamma<3$ is sub optimal. In fact the relative average income $\left\langle\pi_{\gamma=2.5}\right\rangle /\left\langle\pi_{\mathrm{NE}}\right\rangle$ decreases sharply starting from $(T-S)_{c}^{\prime}$ and reaches a minimum when the distance between the QS solution and the NE equilibrium is maximal (for $(T-S)^{\prime}=0.5$ ). Instead for $\gamma>3$ the average payoff coincides with the NE one. Since the average payoff/utility decreases, the collective adoption of strategy $\mathbf{T}$ observed at the phase transition can be regarded as irrational from Expected Utility Theory. Such irrational behaviour is due to the presence of nodes with high degree (hubs) which, for $\gamma<3$, implies a divergent second moment of the co-membership degree distribution [70, 71]. Such hubs can trigger a strategy change in their many co-members, driving the system out of the NE. Simulations for different values of parameters $0 \leq \beta, \zeta \leq 1$ show no appreciable difference with respect to the results in Fig. 1. However, as shown in the SM [30], the synergistic parameter $\beta$ plays a role in the distribution of the average payoff as a function of the group size. In particular, when $\gamma<3$, the groups of all sizes share the same loss of income for $\beta=0$, while for $\beta>0$ only small groups are affected by a loss of income. Not only the average payoff, but also the strategy adoption probability $z_{q}^{*}$ shows a nontrivial dependence on the group sizes. Fig. 2 displays $z_{q}^{*}$ obtained through numerical simulations under simple majority (panels (a),(b)) and two-third majority (panels (c),(d)) decision schemes. In particular, in the two left panels we report $z_{q}^{*}$ as a function of group size $q$. The curves are level curves obtained for different values of $z_{i \in q}$, the fraction of decision makers in state $\mathbf{t}$ among the members of all groups of size $q$, that is the average individual risk propensity of group members. From the definition of $z_{i \in q}$ it follows that, for a given level curve, if $z_{q}^{*}>z_{i \in q} \equiv z_{q=1}^{*}$ (or vice versa $z_{q}^{*}<z_{i \in q} \equiv z_{q=1}^{*}$ ) the average risk propensity of the groups is higher (lower) than the average individual risk propensity of their members, see SM [30]. Thus, the data marked as triangles in panels (a) and (c) show choice shift effects towards strategy $\mathbf{T}$ (risky shift), while squares display a shift towards the safer strategy. The transition between the risky and safe group shift phase occurs at values of $z_{q}^{*}$ equal to the fraction of (a)
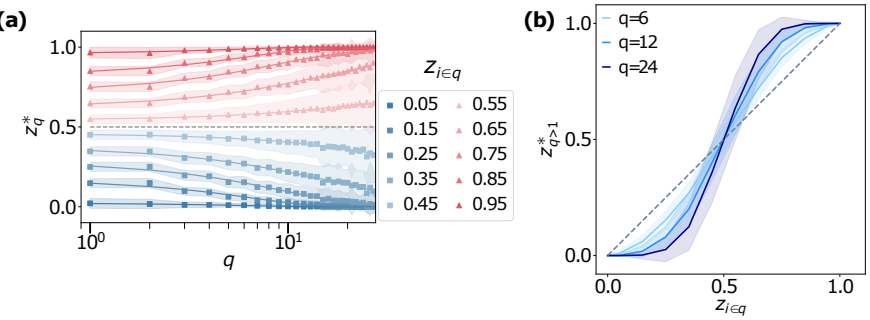

(c)
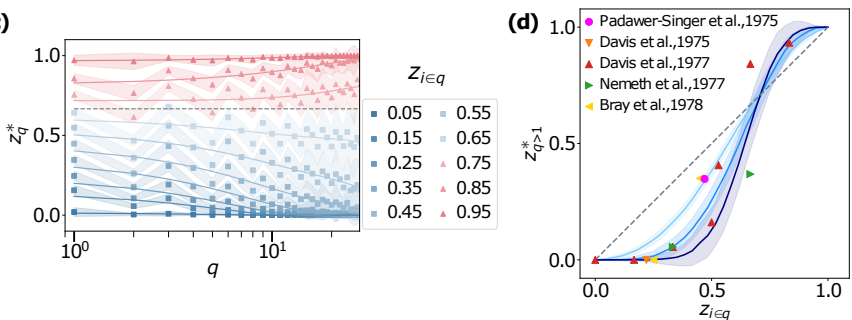

FIG. 2. Choice shift under simple majority (a), (b) and two-third majority (c),(d) decision schemes. In (a), (c), the simulated QS distribution $z_{q}^{*}$ as a function of group size. The grey dotted lines separate risky and safe group shifts. In (b), (d), $z_{q}^{*}$ for groups of size 6, 12 and 24 as a function of $z_{i \in q}$, risk propensity of the members of groups of size $q$. The colored dots in (d) represents empirical data on trial juries from Refs. [25-29]. Shaded areas are the standard deviations. [Parameters: $\beta=0, \zeta=0, \theta=0.01, \lambda=2.5, \nu=2.5$ ].

group members needed to agree the group strategy, respectively $z_{q}^{*}=1 / 2$ and $z_{q}^{*}=2 / 3$. However, independently from the decision scheme adopted, our model shows that the choice shift effect increases with the group size. This is somewhat counter-intuitive, since one would expect that the larger is the group the less probable are extreme collective decisions. We compared the predictions of our model to data from empirical studies about group choice shifts observed in trial juries [25-29] (see SM [30]). To do this, in panel (b) and (d) we plot $z_{q}^{*}$ as a function of $z_{i \in q}$, where the curves are level curves for different sizes $q$. The empirical data (dots), obtained with trial juries of size 6 , are better reproduced by the model with a two-third majority decision scheme. This is in agreement with the observation that a two-third majority scheme is spontaneously adopted by real-world trial juries [26, 39].

In conclusion, our evolutionary game model reproduces, without any ad hoc behavioral assumption, the shifts observed both at a local and a global scale in choice dilemmas, and links them to the structure of the underlying higher-order networks [40]. Our results can also explain how and why radical behaviours can emerge when decisions are taken in groups. Given that co-membership, group size and hyperdegree distributions are easily measurable quantities, our model can provide useful indications on upcoming radical and potentially dangerous group behaviours in online social platforms and other real-world systems, such as financial markets. Our work opens new paths for future research, such as the analytical characteri- 
zation of the observed phase transition and the systematic exploration of the range of possible applications.

We warmly thank Lucas Lacasa and the three anonymous reviewers for their helpful comments and suggestions.

[1] J. A. F. Stoner, A comparison of individual and group decisions involving risk, Ph.D. thesis, Massachusetts Institute of Technology (1961).

[2] N. Kogan and M. A. Wallach, J. Exp. Soc. Psychol. 3, 75 (1967).

[3] K. Eliaz, D. Ray, and R. Razin, Am. Econ. Rev. 96, 1321 (2006).

[4] J. Von Neumann and O. Morgenstern, Theory of games and economic behavior (Princeton University Press, 1944).

[5] M. J. Machina, J. Econ. Perspect. 1, 121 (1987).

[6] C. Starmer, J. Econ. Lit. 38, 332 (2000).

[7] J. H. Davis, Organ. Behav. Hum. Decis. Process. 52, 3 (1992).

[8] W. Edwards, Am. J. Psychol. 67, 56 (1954).

[9] J. H. Davis, N. Kerr, M. Sussmann, and A. K. Rissman, J. Pers. Soc. Psychol. 30, 248 (1974).

[10] T. Gärling, E. Kirchler, A. Lewis, and F. Van Raaij, Psychol. Sci. Public Interest 10, 1 (2009).

[11] S. Bikhchandani and S. Sharma, IMF Staff papers 47, 279 (2000).

[12] R. W. Sias, Rev. Financ. Stud. 17, 165 (2004).

[13] T. Lux, Econ. J. 105, 881 (1995).

[14] M. A. Wallach, N. Kogan, and D. J. Bem, J. Abnorm. Psychol. 65, 75 (1962).

[15] M. A. Wallach, N. Kogan, and D. J. Bem, J. Abnorm. Psychol. 68, 263 (1964).

[16] T. Kameda and R. Hastie, Herd behavior, in Emerging Trends in the Social and Behavioral Sciences (American Cancer Society, 2015) pp. 1-14.

[17] R. B. Zajonc, R. J. Wolosin, M. A. Wolosin, and W. D. Loh, J. Exp. Soc. Psychol. 6, 26 (1970).

[18] P. R. Laughlin and P. C. Earley, J. Pers. Soc. Psychol. 42, 273 (1982).

[19] M. Mäs and A. Flache, PLoS One 8, 1 (2013).

[20] J. K. Shin and J. Lorenz, J. Stat. Mech.: Theory Exp. 2010, P06005 (2010).

[21] F. Baumann, P. Lorenz-Spreen, I. M. Sokolov, and M. Starnini, Phys. Rev. Lett. 124, 048301 (2020).

[22] X. Wang, A. D. Sirianni, S. Tang, Z. Zheng, and F. Fu, Phys. Rev. X 10, 041042 (2020).

[23] E. Burnstein and A. Vinokur, J. Exp. Soc. Psychol. 13, 315 (1977).

[24] J. H. Davis, Psychol. Rev. 80, 97 (1973).

[25] J. H. Davis, N. L. Kerr, R. S. Atkin, R. Holt, and D. Meek, J. Pers. Soc. Psychol. 32, 1 (1975).

[26] J. H. Davis, N. L. Kerr, G. Stasser, D. Meek, and R. Holt, Organ. Behav. Hum. Decis. Process. 18, 346 (1977).

[27] C. Nemeth, J. Appl. Soc. Psychol. 7, 38 (1977).

[28] R. M. Bray and A. M. Noble, J. Pers. Soc. Psychol. 36, 1424 (1978).

[29] S. Penrod and R. Hastie, Psychol. Rev. 87, 133 (1980).

[30] See Supplemental Material at [URL] for the details on the hypergraphs construction and numerical simulations, the analogy with the pairwise game of Chicken in the particular case of a population of two groups of the same size, the full derivation of the main equations, the characterization of the stability of the stationary solution of the mean field dynamics and the analytical expression for the trajectory $z(t)$ in the case of $w_{p}=\frac{\theta}{z}$, for a detailed description of the datasets and the level curves in Fig. 2, for a comprehensive investigation of the relationship between the average payoff as a function of the group size and the synergistic parameter $\beta$, which includes Refs. [31-34].

[31] J. Alstott, E. Bullmore, and D. Plenz, PLoS ONE 9, e85777 (2014).

[32] A. Traulsen and C. Hauert, Reviews of Nonlinear Dynamics and Complexity, Vol. 2 (John Wiley and Sons, Ltd, 2009) pp. 25-61.

[33] M. Archetti, J. Evol. Biol. 22, 2192 (2009).

[34] H. J. Kalven and H. Zeisel, The American jury (Boston: Little, Brown, 1966).

[35] A. Barrat, M. Barthelemy, and A. Vespignani, Dynamical processes on complex networks (Cambridge university press, 2008).

[36] R. Pastor-Satorras, C. Castellano, P. Van Mieghem, and A. Vespignani, Rev. Mod. Phys. 87, 925 (2015).

[37] S. N. Dorogovtsev, A. V. Goltsev, and J. F. Mendes, Rev. Mod. Phys. 80, 1275 (2008).

[38] A. Lahno and M. Serra-Garcia, J. Risk Uncertain. 50, $73(2015)$

[39] P. R. Laughlin and A. Ellis, J. Exp. Soc. Psychol. 22, 177 (1986).

[40] F. Battiston, G. Cencetti, I. Iacopini, V. Latora, M. Lucas, A. Patania, J.-G. Young, and G. Petri, Phys. Rep. 874, 1 (2020).

[41] I. Iacopini, G. Petri, A. Barrat, and V. Latora, Nat. Commun. 10, 2485 (2019).

[42] A. Traulsen and M. A. Nowak, Proc. Natl. Acad. Sci. U.S.A. 103, 10952 (2006).

[43] U. Alvarez-Rodriguez, F. Battiston, G. F. de Arruda, Y. Moreno, M. Perc, and V. Latora, Nat. Hum. Behav. 5 pp. 586-595 (2021).

[44] A. Rapoport and A. M. Chammah, Am. Behav. Sci. 10, 10 (1966).

[45] E. Estrada and J. A. Rodríguez-Velázquez, Physica A 364, 581 (2006).

[46] J.-L. Guillaume and M. Latapy, Physica A 371, 795 (2006).

[47] M. E. J. Newman, Phys. Rev. E 64, 016131 (2001).

[48] S. Milojević, Proc. Natl. Acad. Sci. U.S.A. 111, 3984 (2014).

[49] R. L. Axtell, Science 293, 1818 (2001).

[50] X. Gabaix and Y. M. Ioannides, in Handbook of regional and urban economics, Vol. 4 (Elsevier, 2004) pp. 23412378.

[51] P. Minasandra and K. Isvaran, Behaviour 157, 541 (2020).

[52] H.-S. Niwa, J. Theor. Biol. 224, 451 (2003).

[53] L. Muchnik, S. Pei, L. C. Parra, S. D. Reis, J. S. Andrade Jr, S. Havlin, and H. A. Makse, Sci. Rep. 3, 1 (2013).

[54] N. Perra, B. Gonçalves, R. Pastor-Satorras, and A. Vespignani, Sci. Rep. 2, 469 (2012).

[55] R. Hastie and T. Kameda, Psychol. Rev. 112, 494 (2005).

[56] J. I. Bulow, J. D. Geanakoplos, and P. D. Klemperer, J. Political Econ. 93, 488 (1985).

[57] B. R. Bruns, Games 6, 495 (2015).

[58] J. Y. Campbell, T. Ramadorai, and B. Ranish, Am. Econ. 
Rev. Insights 1, 225 (2019).

[59] L. M. A. Bettencourt, J. Lobo, D. Helbing, C. Kühnert, and G. B. West, Proc. Natl. Acad. Sci. U.S.A. 104, 7301 (2007).

[60] It is worth to notice that an equivalent formulation of this hypothesis is that the group payoff is a function of the group size:

$$
\begin{aligned}
\pi^{(g)}(\mathbf{S}) & =\frac{S}{\left(q^{(g)}\right)^{\zeta}} \\
\pi^{(g)}(\mathbf{T}) & =\left\{\begin{array}{l}
\frac{W}{\left(q^{(g)}\right)^{\zeta}}, \text { with probability } w_{p} \\
\frac{L}{\left(q^{(g)}\right)^{\zeta}}, \text { with probability } 1-w_{p}
\end{array}\right.
\end{aligned}
$$

and that each group member always earns the whole group payoff. We will make use of this interpretation to write the time evolution of the dynamics at the group level, through a coarse-grained approach.

[61] J. Hofbauer and K. Sigmund, Bull. Am. Math. Soc. 40, 479 (2003).

[62] R. Sander, G. Costa, and S. Ferreira, Phys. Rev. E 94, 042308 (2016).
[63] M. de Oliveira and R. Dickman, Phys. Rev. E 71, 016129 (2005).

[64] A. Traulsen, J. C. Claussen, and C. Hauert, Phys. Rev. Lett. 95, 238701 (2005).

[65] A. Traulsen, J. C. Claussen, and C. Hauert, Phys. Rev. E 74, 011901 (2006).

[66] J. Grilli, G. Barabas, M. J. Michalska-Smith, and S. Allesina, Nature 548, 210 (2017).

[67] P. Taylor and L. Jonker, Math. Biosci. 40, 145 (1978).

[68] We tested different power-law distributions with $3<\gamma<$ 4, delta distributions (constant degree) and Poisson's distributions, finding no appreciable difference in the results.

[69] S. C. Ferreira, C. Castellano, and R. Pastor-Satorras, Phys. Rev. E 86, 041125 (2012).

[70] M. E. J. Newman, Networks: An Introduction (Oxford University Press, 2010).

[71] V. Latora, V. Nicosia, and G. Russo, Complex Networks: Principles, Methods and Applications (Cambridge University Press, 2017). 